\section{New Interventional Stroke Trials}

\author{
Martin Bendszus ${ }^{1}$. Jens Fiehler ${ }^{2}$. Götz Thomalla ${ }^{3}$
}

Published online: 14 February 2019

(c) Springer-Verlag GmbH Germany, part of Springer Nature 2019

Endovascular treatment of acute stroke due to large vessel occlusion is undoubtedly extremely effective and nowadays the treatment of choice; however, looking at inclusion criteria of the large randomized clinical trials only highly selected patient populations were treated to demonstrate efficacy in these cohorts. In particular, patients with a poor overall prognosis, e.g. already large infarct reflected by a low Alberta stroke program early CT score (ASPECTS) at presentation or a high likelihood of a benign spontaneous course, e.g., low National Institutes of Health stroke score (NIHSS) at presentation, have either been excluded or are underrepresented in these studies. How should these patients now be handled in clinical practice? Can underpowered post hoc subgroup analysis of trials or observational single center studies be relied on to answer these questions? In the past, unexpected results were often experienced when data from non-randomized studies where later contradicted in randomized clinical trials. Are patients with large infarct cores at presentation really being helped or are the numbers of patients with high disability and a low quality of life just being increased? Moreover, the additional expenses spent on these procedures have to be justified. Likewise, are the outcomes in patients with a very low initial NIHSS compared to the spontaneous course really being improved or are they being exposed to unnecessary risks in view of the possible, although luckily rare complications associated with the intervention?

Martin Bendszus

Martin.Bendszus@med.uni-heidelberg.de

1 Abteilung für Neuroradiologie, Universitätsklinikum Heidelberg, Im Neuenheimer Feld 400, 69120 Heidelberg, Germany

2 Klinik und Poliklinik für Neuroradiologische Diagnostik und Intervention, Universitätsklinikum Hamburg-Eppendorf, Haus Ost 22 (O 22), Martinistr. 52, 20246 Hamburg, Germany

3 Kopf- und Neurozentrum, Klinik und Poliklinik für Neurologie, UKE Hamburg, Hamburg, Germany
To address some of these issues the efficacy and safety of thrombectomy in stroke with extended lesion and extended time window: a randomized, controlled trial (TENSION) was started in 2018 as a randomized controlled trial to assess if patients with a low ASPECTS (3-5) in an extended time window (up to $12 \mathrm{~h}$ after symptom onset) treated with thrombectomy have a better outcome than with best medical treatment alone [1]. Up to 665 subjects from 40 sites in 8 European countries will be included over 3 years. Key inclusion criteria include occlusion of the terminal internal carotid artery (ICA) or M1 segment, ASPECTS 3-5 at presentation on unenhanced computed tomography (CT) or diffusion-weighted imaging (DWI), moderate to severe stroke treatable within $12 \mathrm{~h}$ after symptom onset, age $18-80$ years and absence of severe stenosis in the target vessel. The primary endpoint is functional outcome assessed by the modified Rankin scale (MRS) at 90 days (shift analysis). Additionally, secondary endpoints include the rates of good and moderate outcomes as well as the rates of early neurological improvement and decompressive craniectomy. Moreover, patient-reported health status, quality of life and costs of treatment will be assessed at 12 months. The TENSION study is an investigator initiated trial and receives funding from the European Union's Horizon 2020 research and innovation program.

Conflict of interest M. Bendszus, J. Fiehler and G. Thomalla declare that they have no competing interests.

\section{References}

1. Bendszus M, Bonekamp S, Berge E, Boutitie F, Brouwer P, Gizewski E, Krajina A, Pierot L, Randall G, Simonsen CZ, Zeleňák K, Fiehler J, Thomalla G. A randomized controlled trial to test efficacy and safety of thrombectomy in stroke with extended lesion and extended time window. Int J Stroke. 2018;29:1747493018798558. https://doi.org/10.1177/1747493018798558. 